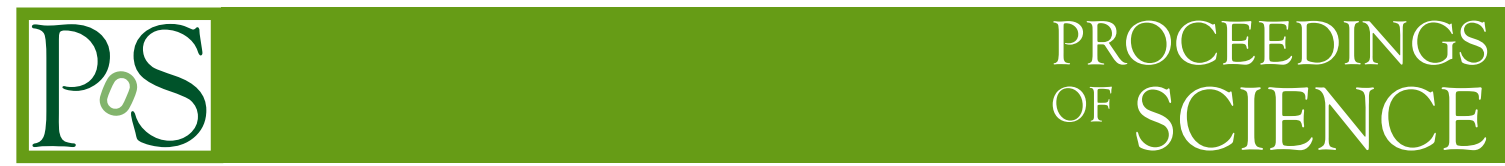

\title{
A study of detector options for eRHIC
}

\author{
Matthew Lamont for the EIC Science Task Force \\ Brookhaven National Lab \\ E-mail: macl@bnl.gov
}

\begin{abstract}
The proposal to build a machine at BNL for Deep Inelastic Scattering on hadrons and nuclei for a wide range of energies provides a number of challenges in order to extract the role of gluons in both nucleons and nuclei. In this paper, I will summarise the efforts and challenges of the BNL EIC Science Task Force in designing a detector to be installed in an unused interaction point of the RHIC ring together with ongoing studies assessing the capabilities and limitations of the current large heavy-ion detectors (STAR and PHENIX) to be able to run in conjunction for e+p/A physics in a staged eRHIC proposal.
\end{abstract}

XVIII International Workshop on Deep-Inelastic Scattering and Related Subjects April 19 -23, 2010

Convitto della Calza, Firenze, Italy 


\section{Introduction}

One of the embodiments of the vision for the future of QCD physics at Brookhaven National $\mathrm{Lab}$ (BNL) is the building of an electron ring which, in conjunction with the currently operating Relativistic Heavy-Ion Collider (RHIC), will allow for electron-ion collisions over a wide range of energies. One of the striking capabilities will be the ability to provide both polarised electrons and protons (and light ions) allowing for detailed investigations of the spin structure of the nucleon. With electron energies ranging from $5-30 \mathrm{GeV}$ and proton (heavy-ion) energies from 50-325 GeV (5-130 GeV/A), a wide range of physics measurements will be possible in understanding the gluon contribution to QCD. One of the topics at the forefront of this frontier is the study of saturation at low- $x$, which may be achievable for the first time by studying DIS in nuclei. For a fuller review of this topic, please read elsewhere in these proceedings [1].

In this paper, I will outline the efforts of the EIC Science Task Force at BNL in designing a detector for eRHIC, as well as the capabilities of the current RHIC detectors (PHENIX and STAR) for contributing to the science measurements at eRHIC. I will not go into great details of the eRHIC machine as this was presented elsewhere at this conference [2].

\section{The capabilities of STAR and PHENIX running in the eRHIC era}

Of the four large experiments associated with RHIC, the two largest (STAR and PHENIX) are still in operation. As it is possible that the heavy-ion programme may extend beyond the start of collisions at eRHIC, it is a useful study to see whether STAR and/or PHENIX have the capability to meaningfully contribute to the e+p/A science at eRHIC.

The STAR and PHENIX experiments employ very different philosophies for heavy-ion collisions. PHENIX is primarily a lepton detector with some capabilities for hadrons but, crucially, it was built to be a fast-readout detector and as such, has only limited acceptance in phase-space. Due to these limitations in acceptance, it is difficult to see a role for PHENIX in its current configuration in the e+p/A mode of operations. However, PHENIX has ambitious ideas for upgrades which may change this conclusion in the fullness of time.

STAR, on the other hand, was built to have a large acceptance and concentrate on hadronic signatures, but with some leptonic capabilities. For this, STAR has a barrel electro-magnetic calorimeter for $|\eta|<1.0$, an endcap electro-magnetic calorimeter for $1.1<\eta<2.0$ and, installed as part of a programme of upgrades, a forward meson spectrometer for $2.3<\eta<4$.0. Figure 1 shows a study of the coverage in $x$ and $Q^{2}$ of the different detectors in STAR for measuring either the scattered lepton or the jet. The figures show the acceptances dependent on whether the hadron beam is in the blue beam pipe (clockwise) or yellow beam pipe (anti-clockwise), something which has yet to be determined and is pivotal in any contribution STAR can make to this effort due to the asymmetrical acceptance of its calorimetry. However, as with PHENIX, any STAR upgrades in the near future may increase the suitability of STAR to run in the e+p/A mode of eRHIC operations.

\section{A purpose-built eRHIC detector}

Although there is a lot of promise for the STAR detector running in the e+p/A mode of eRHIC operations, it is not a detector primarily designed for running in this mode. As such, there are many 

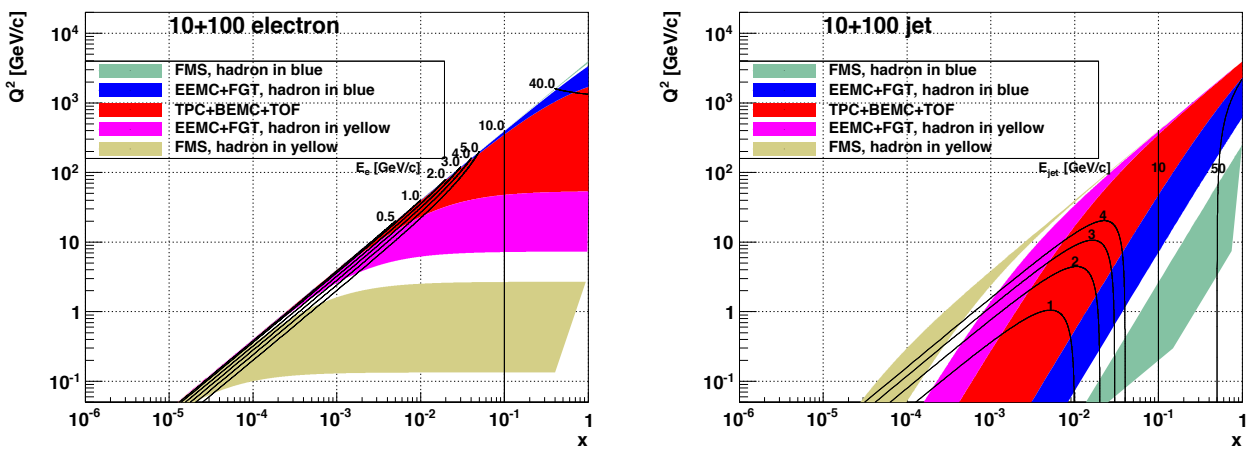

Figure 1: The coverage in $x$ and $Q^{2}$ of the different detectors in STAR for $10+100 \mathrm{GeV} e+h$ collisions for measuring the scattered $e^{-}$(left) and jet (right) respectively. In each case, the two different options for the hadron beam being in the blue beam pipe (clockwise) or yellow beam pipe (anti-clockwise) are given.

holes in the capabilities of the detector which would need to be confronted. For example, one of the surprising outcomes of the HERA physics programme of $e+p$ collisions was the large fraction of diffractive events, contributing $10-15 \%$ of the total cross-section. One of the key signatures of these diffractive events was an intact proton travelling at nearly beam energies, together with a gap in rapidity before some final-state particles were produced at mid-rapidity. However, the detectors (H1 and ZEUS) were not optimised for this important physics and indeed were not fully hermetic, with large gaps at forward rapidities. This was a crucial factor in their inability to measure the scattered proton, which was only achievable after a programme of upgrades.

Therefore, any running of STAR and PHENIX in eRHIC operations can only be seen as being complimentary to a full purpose-built detector. This will have the advantage that it is built specifically for $e+p /$ A collisions and can be designed on the back of new detector technologies, two decades after STAR and PHENIX were proposed.

\subsection{Detector requirements}

When designing a detector, a number of requirements are placed on the design and composition depending upon the physics being measured. For example, in order to measure diffractive physics at low $x$, it is desirable to have very forward detectors at small angles with respect to the beam line. These detectors are referred to as "Roman Pots" and are successfully used in the pp2pp experiment and are integrated into STAR where they are used for elastic $p+p$ collisions [3].

Other requirements are that the same detector should be able to measure all processes, such as inclusive ( $e p \rightarrow e^{\prime} \mathrm{X}$ ), semi-inclusive ( $e p \rightarrow e^{\prime} \mathrm{X}+$ hadrons) and exclusive (e.g. $e p \rightarrow e^{\prime} p+J / \Psi$ ) reactions. It is also crucial to have particle identification $(e, \pi, k, p, n)$ over a wide range of momentum and scattering angles. It is also important to measure heavy flavour particles (those comprised of charm and beauty quarks) well. To achieve this, it is important to have good secondary vertex resolution. The requirements for $e+p$ and $e+\mathrm{A}$ collisions are very similar, the only additional complication in $e+\mathrm{A}$ collisions arises from the need to tag the struck nucleus compared to the struck nucleon. 


\subsection{Detector design}

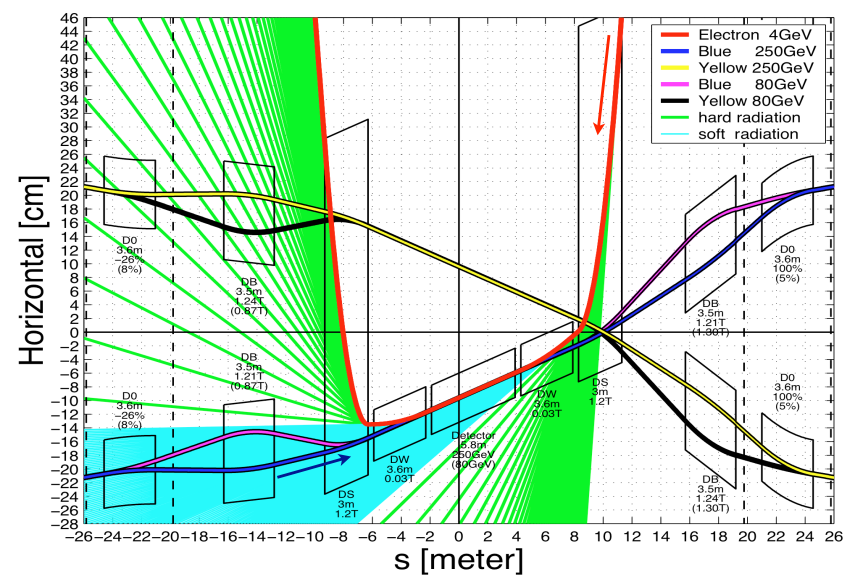

Figure 2: A design of the interaction region for eRHIC. The electron beam is in the red beam pipe (coming from the upper right) and the ion beam is in the blue beam pipe (coming from the lower left).

One of the first things which must be accomplished when designing a new detector is a design of the interaction region (IR) as this places limits on the physical size of the detector. Figure 2 shows a design produced by the Collider-Accelerator Department (C-AD) which has the electron beam in the red beam pipe (coming from the upper right) and the ion beam in the blue beam pipe (coming from the lower left). When electrons are bent in a magnetic field, they emit Bremsstrahlung radiation and it is imperative that any of this radiation does not go through the detector. In Figure 2, no additional shielding has been put in the design but even without any of the shielding in place, none of the hard radiation will enter the detector. This has been designed for the IR at 12 o'clock at RHIC. Both the 12 o'clock and 2 o'clock IRs have space for a detector but it has been determined that the 12 o'clock region is larger and will require less civil construction before a detector is built.

As can be seen in the figure, the IR requirements leave a $6 \mathrm{~m}$ space in the longitudinal direction for a detector before the magnets which affect the beam come into place. This places constraints on the detector design. A sketch of a possible detector is shown in Figure 3 which would fit into this space. In the barrel region at mid-rapidity, it consists of a solenoidal field with Si tracking around the interaction point itself, followed by Cherenkov detectors for particle identification and then both electro-magnetic and hadronic calorimeters. There are further trackers and calorimeters at forward rapidities, but this time, the magnetic field is a dipole. This is just a starting point and other technologies (such as time-of-flight) are under active investigation.

Progress has been made in implementing this design into the GEANT framework, where it is possible to pass MC generated events (using such packages as Pythia (for DIS events) and RAPGAP (for diffractive events)) through the detector setup. It is possible to then take this data and calculate quantities such as momentum resolution and energy loss across a detector. This is an iterative process which leads to an optimisation of the detector design. It is particularly convenient as it is optimised to accept input from any event generator and is fairly quick, leading to a fast 


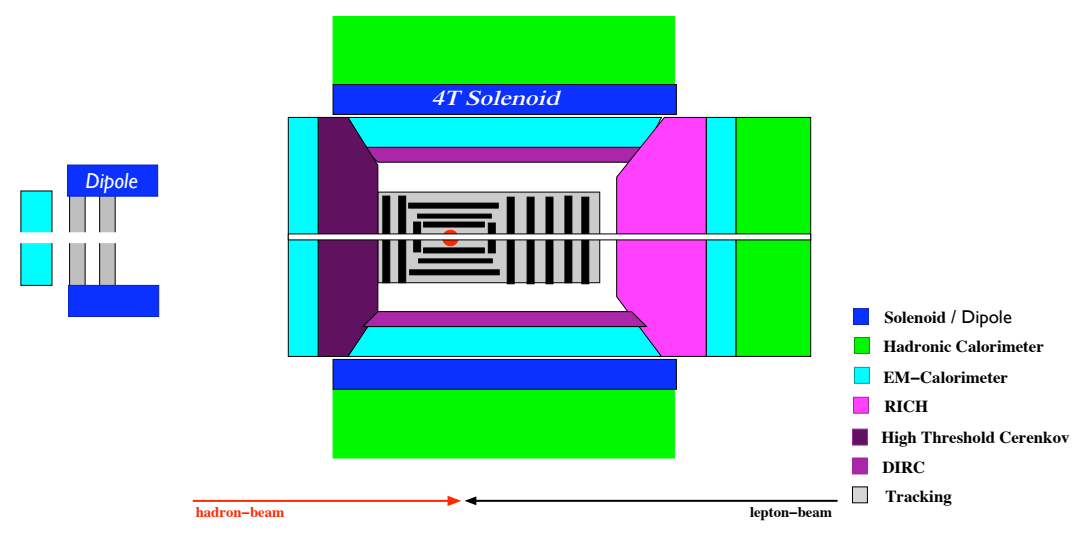

Figure 3: A design of the interaction region for eRHIC. The electron beam is in the red beam pipe (coming from the upper right) and the ion beam is in the blue beam pipe (coming from the lower left).

turn-around time on the design. Figure 4 shows an example of a Pythia event being passed through the detector simulation.

Figure 4: An $e+p$ event, generated with Pythia, being passed through an implementation of the detector design, written in GEANT 3.

\section{References}

[1] M. A. C. Lamont for the EIC Science Task Force, these proceedings.

[2] I. Ben-Zvi, these proceedings.

[3] S. Bültmann et al.Nucl. Instrum. and Meth. in Phys. Research A535, 415 (2004). 